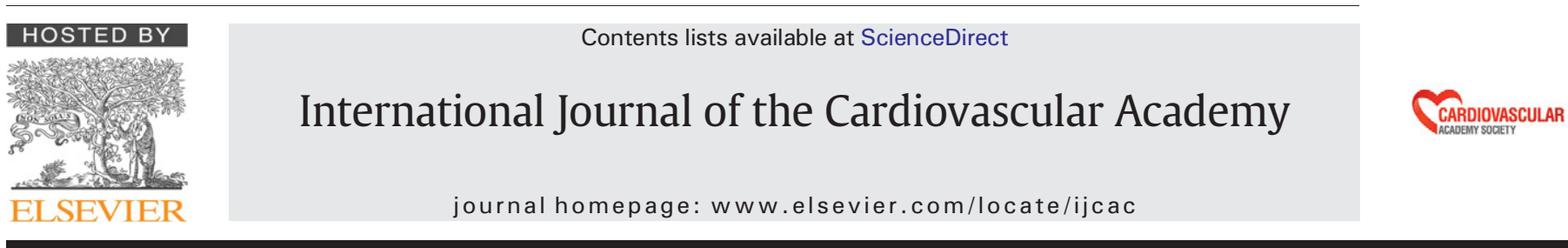

Review

\title{
NOACs and routine coagulation assays. How to interpret?
}

\author{
Ebru Ipek Turkoglu \\ Department of Cardiology, Izmir Kemalpasa Devlet Hastanesi, Izmir, Turkey
}

\section{A R T I C L E I N F O}

\section{Article history:}

Received 10 October 2015

Accepted 22 October 2015

Available online 12 November 2015

\section{Keywords:}

NOAC

PT

aPT

\begin{abstract}
A B S T R A C T
Since the non-vitamin K antagonist oral anticoagulants (NOACs) have been introduced to clinical practice, there is a conflict on coagulation tests, which have been used to monitor the anticoagulation effect of vitamin $\mathrm{K}$ antagonists (VKAs) and unfractionated heparin (UFH). NOACs have alternative modes of action. They react differently than VKAs and UFH and therefore conventional coagulation tests are not suitable to monitor their anticoagulant effect. The interactions between NOACs and routine coagulation tests are discussed in this review.

(C) 2015 The Society of Cardiovascular Academy. Production and hosting by Elsevier B.V. All rights reserved. This is an open access article under the CC BY-NC-ND license (http://creativecommons.org/licenses/by-nc-nd/4.0/).
\end{abstract}

\section{Contents}

Introduction . . . . . . . . . . . . . . . . . . . . . . . . . . . . . . . . . . . . . . . . . 41

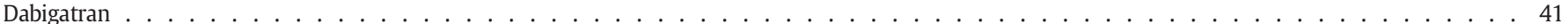

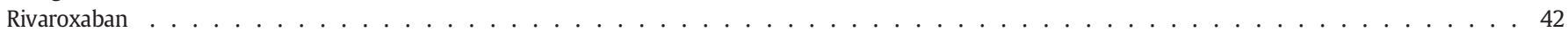

Apixaban . . . . . . . . . . . . . . . . . . . . . . . . . . . . . . . . . . . . . . . . 42

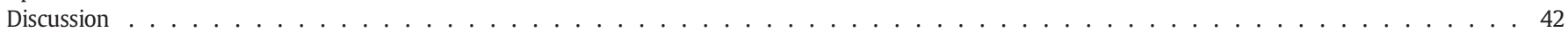

Conclusion . . . . . . . . . . . . . . . . . . . . . . . . . .....................42

References .. . . . . . . . . . . . . . . . . . . . . . . . . . . . . . . . . . . . . . . . 42

\section{Introduction}

Many anticoagulant agents are available for prevention and treatment of thromboembolic disorders such as the warfarin derivatives (vitamin K antagonists-VKAs), unfractionated heparin (UFH) and low molecular weight heparins (LMWHs) ${ }^{1,2}$ In the last decade a new class of anticoagulant drugs has been developed for clinical use. The nonvitamin $\mathrm{K}$ antagonist oral anticoagulants (NOACs) are orally active and inhibit coagulation serine proteases selectively and specifically. ${ }^{3}$ Unlike VKA and UFH, ${ }^{4,5}$ the NOACs such as dabigatran (direct thrombin inhibitor), rivaroxaban, apixaban and edoxaban (all direct Factor Xa inhibitors) do not require routine coagulation monitoring because they have a rapid onset of action, their therapeutic window is wide and their pharmacokinetics and pharmacodynamics are predictable. ${ }^{6,7}$ However in some clinical situations the need for assessment of the anticoagulant effect of these new agents might arise, which requires an understanding of the different mechanism of action of each agent and their implications when interpreting routine coagulation tests are necessary. ${ }^{1}$ The most commonly used clot-based tests are prothrombin time (PT)/international normalized ratio (INR) and activated partial thromboplastin

Peer review under responsibility of The Society of Cardiovascular Academy. time (aPTT). ${ }^{1}$ PT is the time in seconds for plasma to coagulate after addition of calcium and an activator of the extrinsic pathway of the coagulation cascade as thromboplastin. ${ }^{8}$ PT is mostly used to monitor VKAs which inhibits factors II, VII and X..$^{9}$ Because of varying sensitivities of the available thromboplastin agents, PT is converted to INR through a mathematical formula that includes the International Sensitivity Index (ISI) of the thromboplastin reagent used. ISI is obtained from the manufacturer. ${ }^{10}$ After the calculation, INR can be interpreted without regard to the thromboplastin reagent. ${ }^{8}$ While aPTT is similar to PT, aPTT reflects the presence and activity of factors II, V, VIII, XII and fibrinogen. ${ }^{8}$ Therefore, the test is used to measure the overall function of the intrinsic coagulation pathway. ${ }^{1}$ However these tests do not react in the same way for NOACs and may be misinterpreted by nonexpert clinicians. ${ }^{1,3}$

\section{Dabigatran}

The PT is relatively insensitive to dabigatran and the PT reagent sensitivity is extremely variable. ${ }^{11,12}$ The INR/ISI calculation which is used for warfarin derivatives is not suitable for dabigatran. The contradiction between PT results is increased by point of care-derived INR results. ${ }^{13,14}$ 
The aPTT is more sensitive to the anticoagulant effect of dabigatran and shows a curvilinear dose response with an increase at low concentrations and linearity at high concentration. ${ }^{12,15}$ The aPTT results are influenced by coagulometers and reagents and need to be evaluated carefully. In the nationwide Belgian survey, Actin FSL is found the least sensitive among aPTT reagents. The other reagents (Actin FS, APTT-SP, STA-CK Prest, STA-Cephascreen, STA-PTT A, SynthASil and TriniCLOT aPTT HS) used in the survey show slightly higher sensitivities. ${ }^{21}$ A normal aPTT can presumably exclude a therapeutic intensity of dabigatran but cannot exclude some degree of anticoagulation caused by dabigatran. ${ }^{3}$ If the aPTT level at trough exceeds two times the upper limit of normal, this can be considered as a higher risk of bleeding. ${ }^{16}$

\section{Rivaroxaban}

Rivaroxaban plasma levels in healthy subjects correlate closely with the inhibition of Factor Xa activity and prolongation of PT and aPTT. ${ }^{17,18}$ The PT is more sensitive for rivaroxaban. There is a linear concentration-response correlation but because of the high variability between PT reagents, up to three-fold difference between them can be observed. ${ }^{3,19}$ The INR calculation increases the contradiction between PT results (nearly four-fold), because ISI are calculated for VKAs, and therefore INR results should not be used. ${ }^{1,3,19}$ A normal PT level cannot exclude some degree of anticoagulation effect but a normal PT level achieved with most reagents excludes a therapeutic intensity of rivaroxaban. ${ }^{3}$ With the thromboplastin reagents, neoplastin and neoplastin plus, the PT is influenced in a dose-dependent manner with a close correlation to plasma concentrations. ${ }^{16}$ In the Belgian survey, where six different PT reagents were used, the reagents Innovin and Thrombel S were found least sensitive to rivaroxaban and Neoplastin $\mathrm{R}$ was the most sensitive PT reagent to rivaroxaban. ${ }^{21}$ The sensitivity difference was not connected with the source of tissue factor and it may be related to the composition of the reagents. ${ }^{21}$

\section{Apixaban}

Apixaban prolongs PT in human plasma in vitro. ${ }^{20}$ As with rivaroxaban, there is variability in the PT sensitivity between thromboplastin reagents and the INR calculation increases variability. ${ }^{3,19}$ To measure the inhibition of Factor Xa activity gives a better sign of apixaban plasma concentration than a PT test. ${ }^{19}$

Apixaban has been shown to prolong the aPTT in a concentration dependent manner in vitro but the aPTT test is not sensitive to apixaban. ${ }^{1,20}$

\section{Discussion}

In clinical practice NOACs are prescribed at fixed doses and do not require routine coagulation monitoring, but their presence can affect routine coagulation tests especially in high-doses or if the blood sample is taken at the peak plasma concentration level and these may lead to interpret the test results improperly. ${ }^{21}$ NOACs prolong the PT and aPTT in a concentration- and reagent-dependent manner. There is a significant difference in sensitivity of reagents. PT reagents are more influenced by rivaroxaban and apixaban and APTT reagents are more influenced by dabigatran. ${ }^{21}$ In monitoring VKA therapy, PT results are expressed as INR levels commonly in daily clinical practice. However for NOACs, converting PT to INR does not decrease but even increases the variability between reagents and cannot be applied because the conventional INR system was developed to monitor VKAs and correct the varying sensitivities of reagents for VKAs, not for NOACs. ${ }^{21}$

\section{Conclusion}

It is important for clinicians to appreciate the variable effects of individual NOACs on PT and aPTT reagents utilized to avoid erroneous interpretation of results.

\section{References}

1. Samama MM, Guinet C. Laboratory assessment of new anticoagulants. Clin Chem Lab Med 2011;49(5):761-772.

2. Geerts WH, Bergqvist D, Pineo GF, et al. Prevention of venous thromboembolism: American College of Chest Physicians Evidence-based Clinical Practice Guidelines, 8th edition. Chest 2008;133:381S-453S.

3. Baglin T. The role of the laboratory in treatment with new oral anticoagulants. $J$ Thromb Haemost 2013;11(Suppl 1):8-112.

4. Hirsh J, Bauer KA, Donati MB, et al. Parenteral anticoagulants: American College of Chest Physicians Evidence-based Clinical Practice Guidelines, 8th edition. Chest 2008;133:141S-159S

5. Ansell J, Hirsh J, Hylek E, et al. Pharmacology and management of the vitamin K antagonists: American College of Chest Physicians Evidence-based Clinical Practice Guidelines, 8th edition. Chest 2008;133:160S-198S.

6. Samama MM, Gerotziafas GT. Newer anticoagulants in 2009. J Thromb Thrombolysis 2010;29:92-104.

7. Bauer KA. New anticoagulants. Curr Opin Hematol 2008;15:15-509.

8. Miyares MA, Davis K. Newer oral anticoagulants: a review of laboratory monitoring options and reversal agents in the hemorrhagic patient. Am J Health Syst Pharm 2012;69:84-1473.

9. Ageno W, Gallus AS, Wittkowsky A, et al. Oral anticoagulant therapy: antithrombotic therapy and prevention of thrombosis: American College of Chest Physicians Evidence-based Clinical Practice Guidelines, 9th edition. Chest 2012;141:e44S-e88S.

10. Kamal AH, Tefferi A, Pruthi RK. How to interpret and pursue an abnormal prothrombin time, activated partial thromboplastin time and bleeding time in adults. Mayo Clin Proc 2007;82:73-864.

11. Lindahl T, Baghaei F, Blixter IF, et al. Effects on the oral, direct thrombin inhibitor dabigatran on five common coagulation assays. Thromb Haemost 2011;105:8-371.

12. van Ryn J, Stangier J, Haertter S, et al. Dabigatran etexilate - a novel, reversible, ora direct thrombin inhibitor: interpretation of coagulation assays and reversal of anticoagulant activity. Thromb Haemost 2010;103:27-1116.

13. Baruch L, Sherman O. Potential inaccuracy of point-of-care INR in dabigatran-treated patients. Ann Pharmacother 2011;45e40.

14. De Remer CE, Gujral JS, Thornton JW, et al. Dabigatran falsely elevates point of care international normalized ratio results. Am J Med 2011;124:e5-e6.

15. Douxfils J, Mullier F, Robert S, et al. Impact of dabigatran on a large panel of routine or specific coagulation assays. Laboratory recommendations for monitoring of dabigatran etexilate. Thromb Haemost 2012;107:97-985.

16. Heindbuchel H, Verhamme P, Alings $M$, et al. European Heart Rhythm Association Practical Guide on the Use of New Oral Anticoagulants in Patients with Non-valvular Atrial Fibrillation. Europace 2013;15:51-625.

17. Samama MM, Martinoli JL, LeFlem L, et al. Assessment of laboratory assays to measure rivaroxaban - an oral, direct factor Xa inhibitor. Thromb Haemost 2010;103: 25-815.

18. Eriksson BI, Borris L, Dahl OE, et al. Oral, direct factor Xa inhibition with BAY59-7939 for the prevention of venous thromboembolism after total hip replacement. J Thromb Haemost 2006;4:8-121.

19. Barret YC, Wang Z, Frost C, et al. Clinical laboratory measurement of direct factor Xa inhibitors: anti-Xa assay is preferable to prothrombin time assay. Thromb Haemost 2010;104:71-1263.

20. Wong PC, Crain EJ, Xin B, et al. Apixaban, an oral, direct and highly selective Factor Xa inhibitor: in vitro, antithrombotic and antihemostatic studies. J Thromb Haemos 2008;6:9-820.

21. Van Blerk M, Bailleul E, Chatelain B, et al. Influence of dabigatran and rivaroxaban on routine coagulation assays. A nationwide Belgian survey. Thromb Haemost 2015;113(1):64-154. 